\title{
Mobile Wellness Innovation: Qi Gong App to Improve Wellness and Cognitive Resiliency in Older Adults
}

\author{
Colleen McMillan PhD \\ Tony Tin MA, MLS
}

This pilot project explored the utility of a mobile health and wellness app to older adults interested in using low impact exercise as a protective factor against memory and mood loss. While it is known that exercise is a protective factor in preventing further cognitive regression, it is shown that adults 55 and older spend ten hours or more each day sitting or lying down, leaving the latter group even more compromised (Cavill, 2013). The piloting of a health and wellness self management tool through a mobile app featuring the Chinese exercise of Qi Gong represents an innovative, visual and accessible tool that supports daily physical activity while fostering a sense of personal empowerment and enhancing the quality of life.

\section{Revelance of exercise to Canada's aging population}

Canada is facing a dementia epidemic with approximateley 500,000 Canadians experiencing Alzheimers or an associated dementia (Hopkins, 2010). It is the most significant and widespread form of disability among Canadians who are 65 years and older (Hopkins, 2010). By the year 2050 there will be 115 million people world wide afflicted with this progressive disease (Wilkinson, 2010). While there remains no cure, mild to moderate exercise has been clinicially shown to reduce the risk of sunsequent dementia as well as function as a protective factor. Studies demonstrate that modified exercise programs can slow down the decline in healthrelated quality of life among heterogeneous, older persons residing in institutions (Kwak, Um, Son et al., 2008; Dechamps, Diolez, Thiaudière et al., 2012). Equally, if not more important, it supports older adults who prefer to remain in their homes within a familiar community representing an additional protective factor against aged related loneliness and depression.

Aside from the mental health component, increasing the general physical state of this population addresses the most prevalent risk, that of falling. A study conducted in British Columbia found that one in three adults over the age of 65 fall once a year threatening their physical and emotional independence (BC Ministry of Health, 2014). Exercises that strengthen the core as a preventive measure of gait imbalance is key to fall prevention and overall stability in older adults, a health issue that is quickly gaining critical importance. The Center for Disease 
Control and Prevention found that $30 \%$ of persons 65 and older report difficulty walking three city blocks or climbing one flight of stairs, and approximately $20 \%$ require the use of a mobility aid to ambulate (Centers for Disease Control and Prevention, 2009). In a 2006 study of non institutionalized older adults, authors Verghese, LeValley, Hall et al. reported that $35 \%$ of the participants had an abnormal gait posing a risk factor for potential falls.

While past studies demonstrate moderate exercise that is aerobic based shows the most optimal outcomes (Kramer, 2010) it is also know that the majority of residents in institutional care are sedentary at best (Hse, Wang, Chen et al., 2010). Compounded by increasing frailty, aerobic exercise is neither suitable nor recommended for many older institutionalized adults or those who remain in their homes. Cognizant of this physical limitation, our project focuses on the centuries old Chinese practice of Qi Gong, that incorporates gentle breathing and non strenuous stretching and holding exercises for older adults who have minimal to nil fitness levels.

\section{Qi Gong, Wellness and Aging}

Qi Gong is a traditional Chinese mind and body wellness exercise that dates back to the ancient Han Dynasty (206 BC-220 AD). The practice of Qi Gong incorporates gentle breathing and nonstrenuous stretching and holding exercises that follow the 12 major meridians of the body. By doing so, balance is achieved between the Yin and Yang of the body (Chinese Health Qi Gong Association, 2012)

A growing body of published research is emerging that documents the health benefits of Qi Gong. From a western standpoint of evidence based practice, the holistic nature of Qi Gong exercises activate naturally occurring physiological and psychological mechanisms of self-repair and health (Jahnke, Larkey, Rogers et al. 2010). Clinical studies indicate that Qi Gong exercises

can enhance the functions of the respiratorypository, nervous and cardiovascular systems, build up the cellular immune function and the ability to resist senility, and improve body balance (Sancier \& Holman, 2004). Cohen (1999) maintained that the practice of Qi Gong could improve cognitive function in aging and alleviate the symptoms of senile dementia. This claim is supported by other studies that demonstrate Qi Gong can shorten reaction speed, enhance short-term memory and prolong attention abilities, significantly acting as protective factors toward healthy aging (Burgener, Yang, Gilbert, \& Marsh-Yant, 2008; Dong, Kim, Lee et al., 2013) 


\section{Current literature on techology and exercise for older adults}

There is a growing body of evidence based literature correlating the benefits of exercise as both a preventative and management approach with individuals diagnosed with Alzheimers and other dementias. With the exception of music therapy for older adults with memory loss, there is a void in the literature regarding the utility and efficacy of etechnology regarding exercise programing for older adults with early signs of dementia. A major factor contributing to the pervasive lack of research concerns the perception that older adults lack or are uninterested in digital literacy. Authors Melenhorst, Rogers and Caylor (2011) challenged this perception in a study that looked at the experiences and attitudes of older adults dependent upon medical devices (such as self monitoring diabetes devices). The authors found that the population studied were receptive and engaged in technology, contingent upon simple instructions and clear training. In an article titled, "How can technology contribute to the quality of life of older adults?" Rogers and Mynatt (2003) summed up the impetus to explore technology as a viable avenue to improve quality of life by stating "the challenge is to design interfaces that reflect and support ongoing activities of daily living but not be inappropriately intrusive" (p. 26). A later study by Armas, Broderick, Coye et al., (2009) found that older adults wanted to be active stakeholders in the management of their health and wellness.

Literature indicates that this age group is less likely more unlikely to join a gym or travel to a central exercise destination than younger cohorts. A study done at the University of Illinois using a control group found that older adults were more likely to stay involved with a homebased exercise program that they enjoyed and experienced marginal improvement over those who traveled to and exercised at a central location, such as a gym or community centre. The 2013 study also found that those individuals who stayed with the DVD at home exercise program experienced clinically significant improvements pertaining to gait, balance and strength. The study also found that ten minute intervals spread throughout the week was more appealing to older adults as it was easier to attain and resulted in higher satisfaction levels (Traywick, 2014).

The use of a mobile app designed to encourage regular exercise is an area that remains unexplored (Rogers \& Mynatt, 2003). Methods to stimulate neurotransmitters in older adult brains through exercise programs is not new, however, the use of digital mobile technology as a self management tool to encourage exercise is (Center for Technology and Aging, 2009:

Melenhoust, Rogers, \& Caylor, 2001). Current research has found that older adults have higher usage of tablets at $27 \%$ than the general public at $18 \%$, and despite some challenges unique to 
this age group (learning curve, sckeptical attitudes or physical barriers such as arthritis) the older adult population possesses strongly positive attitudes about the benefits of technology in their personal lives, including that of health (Smith, 2014).

\section{Methodology}

\section{Research Design}

The Qi Gong Wellness App is based upon the ancient Chinese practice of Qi Gong which integrates the "three regulations": body focus (posture and movement), breath focus, and mind focus or meditative components (Jahnke, 2002)

The visual scheme of the initial webpage was colour coded to emulate the three colours of a symbol familiar to the majority of this population, being the traffic stop light - green, yellow and red as shown in Figure 1.

Figure 1. Opening Webpage

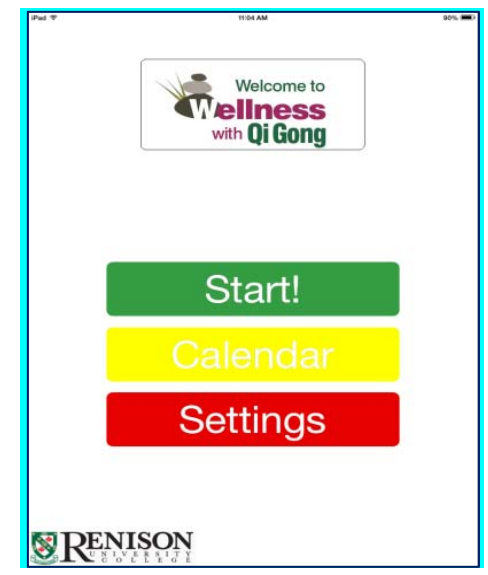

To remain authentic to the origins of Qi Gong, the authors asked for permission from the founder of the Qi Gong Chinese Health Association to incorporate existing exercise videos into the app. The physical demonstration of Qi Gong was provided through nine different videos of 
two minutes each with both the front and back view available to the viewer as demonstrated by Figure 2.

Figure 2. Qi Gong Video Exercise Clip -Front and Back View

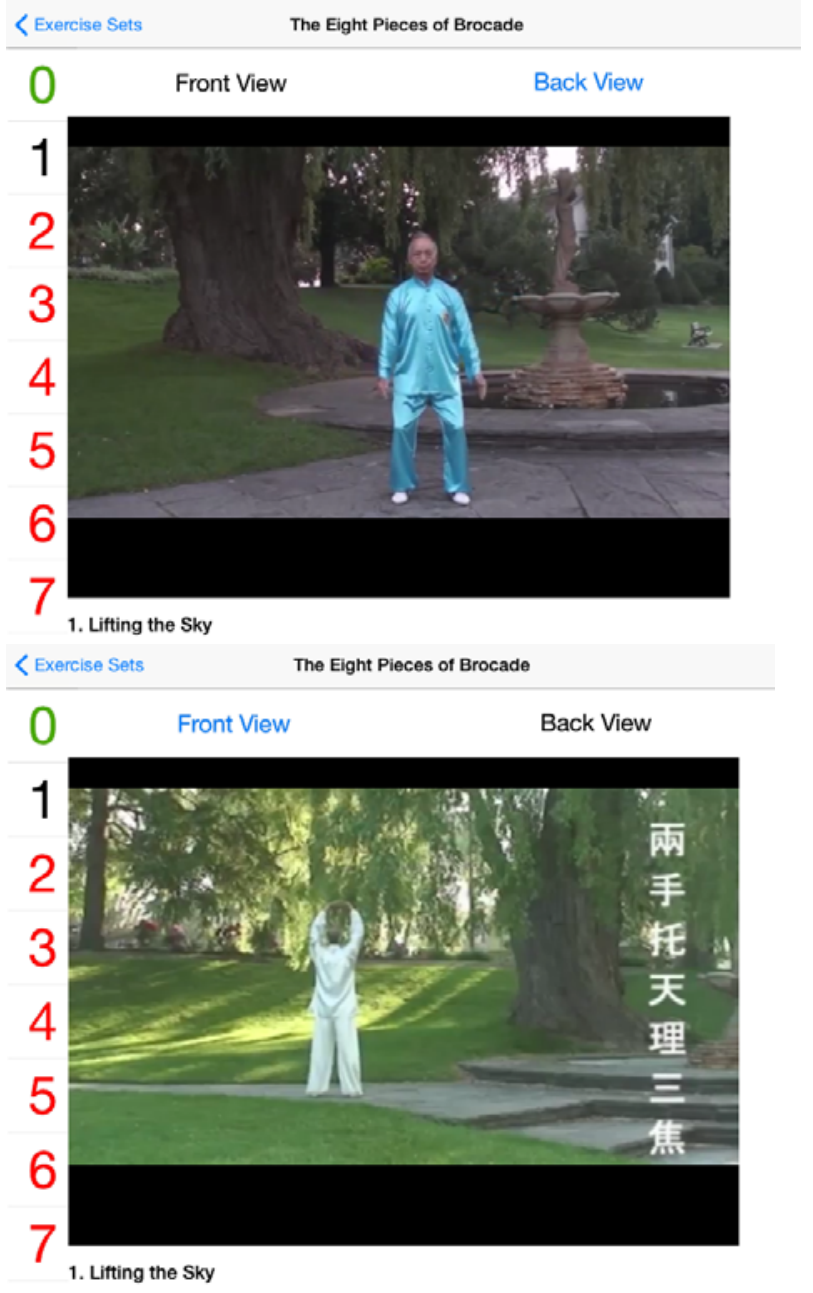

The viewers choose the frequency of the exercises (day and time) by selecting an embedded audio reminder adapted to individual preferences and schedules illustrated by Figure 3 . The user was also prompted to rate their mood before and after the exercises that then was recorded visually for the viewer to monitor the association between mood and exercise in the User Records. The mechanism for mood recording was based upon the UNICEF model of participatory evaluation using a likert scale of 'happy faces' as shown in Figure 4. 
Figure 3. Embedded Audio Reminder

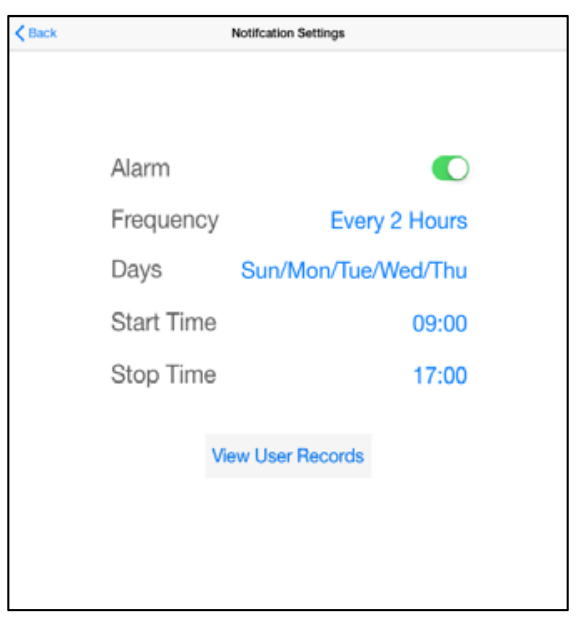

Figure 4. Mood Ranking Scale

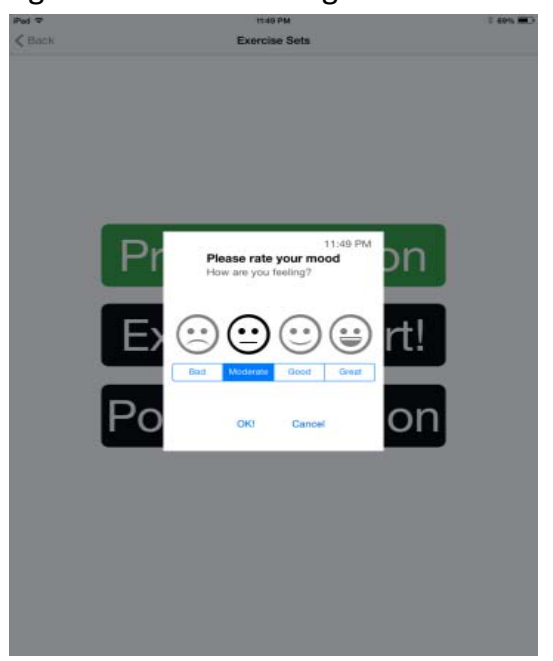


A calendar recorded the mood entered by the participant to visually make the association between their mood and the Qi Gong exercises on the day(s) selected illustrated in Figure 5.

Figure 5. Calendar Recording Baseline of Mood

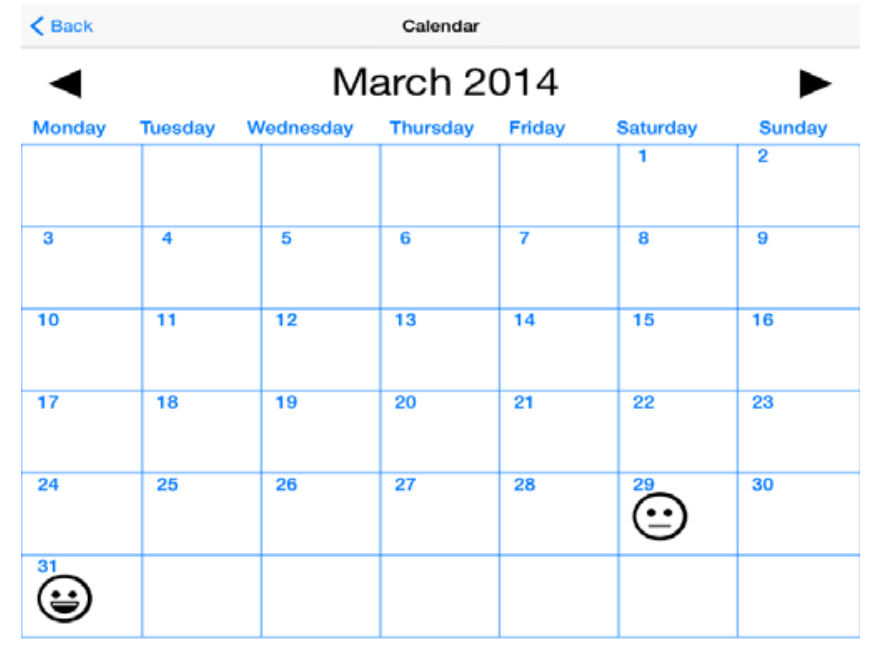

This application operated under the iOS platform and ran on an iPhone/iPad device.

\section{Sample Population}

The described Qi Gong Wellness app was premiered at the Qi Gong Chinese Health Qi Gong Association Conference in Toronto, Canada in May 2014 with an international audience spanning all age groups. Participants attending the conference were invited to participate and to later record their experience with the app on Survey Monkey. A total of 27 individuals volunteered to participate in the pilot study, $\mathrm{X}$ males and $\mathrm{X}$ females.

The participants were asked a series of demographic and experiential questions including;

- age group

- whether they liked the Qi Gong exercises 
- whether they liked the mobile technology of the Wellness App

- if the video was helpful in following the exercise routine

- if the background information was helpful in understanding Qi Gong as an exercise

- if the link between mood and exercise was liked

- whether they would use the Qi Gong Wellness App on a regular basis

- any other comments they wished to add

A five point likert scale was used to collect participant responses. Quantitative analysis was done through descriptive statistics augmented by qualitative thematic coding of opened ended responses. This method was used for purposes of data triangulation. Ethical approvals was applied for and received from the University of Waterloo. As acknowledgement for participating, a draw was held one month after the conference for participants to win one of four gift certificates. A thank you email was sent out to each participant asking if they would consider being on a future Advisory Committee for the next development of the Qi Gong Wellness App.

\section{Findings}

Findings will be shared in the order of the questions posed to the participants.

Based upon the age ranges that attended the Qi Gong Chinese Health Qi Gong Association conference from where the sample size was drawn from, the content featured in the app appeared to be most attractive to the age group of 45 to 59 and 60 to 64 based upon the response rate. Just fewer than 60 percent reported liking the app "very much," the highest ranking on the likert scale. However, the qualitative comments regarding ease and relevancy of the exercises were stated most in the third age category of 70 to 74 years. Comments included "exercises are very doable for my age" and "the app helped me do the exercise in my garden."

The question regarding general likeability of the Wellness App received the overall highest ranking of any survey question with 59.26 percent. What features attributed to this ranking included comments such as "I really liked the exercises" and "convinces me to do such exercises."Other comments spoke to the "innovative" nature of the App. 
The majority of the 21 open ended comments were in response to the question "Did you like the mobile technology of the Wellness App?" with combined percentage points of over 82 (equal ranking of 41.67 was given to the categories of mostly and very much). The comments comprised of what the participants found helpful such as "being able to see both sides" to suggestions on how to enhance the technological aspects such as "if the download of this application is available on smart phones, the mobile Qi Gong will be more popular and be widely used. More people own a smart phone instead of ipad/iphone" and "I am not sure if I'd view the exercises in a mobile device smaller than an Ipad ie. cellphone."

As to whether the videos demonstrating the Qi Gong exercises were helpful in following the routine, participants offered specific improvements. Despite being ranked as "helpful to very helpful" with a combined category percentage of 91.67, several participants asked for "verbal instructions" to accompany the videos, a few asked for background music, but the majority of comments regarded the logistics of the video, such as "I would like to be able to click any of the exercise numbers and watch. Currently, it is only in fixed sequence and no user control for picking up a certain video clip." Variations of these comments were common in the feedback.

More than double the participants found the research behind the Qi Gong Wellness App to be either "helpful" or "very helpful" at 65.21 percent as compared to those who ranked this question as "not at all, somewhat and neutral" at a combined 34.78 percent. Suggestions included providing "an explanation of what Qi Gong is, such as the history and how it is believed to be beneficial" and "more information on how Qi Gong works for my health."

One of the distinguishing features of the Qi Gong Wellness App was the linking of the participant's pre and post mood to the exercises. Almost 70 percent of the participants ranked this feature as being "mostly to very well liked". The link was ranked as "mostly liked" by 43.48 percent followed by "very much liked" by 26.09 percent. One participant suggesting broadening the question to "rate your mood concerning your body fitness/health."

Lastly, the question asked the participants if they were planning to use the Qi Gong Wellness App regularly. A combined total of 61 percent was affirmative, with 39.13 percent responding that they would "most likely" use the app followed by 21.74 percent who said they "definitely" 
would use it. Over half of the participants stated that they would use it, with one participant summing up similar comments with the statement "thanks for creating this app. I will definitely use it regularly" and "hope we can view more Health Qi Gong and Tai Chi in the future."

The most frequent suggestion given by the participants regarded the fixed sequence of the videos, meaning that only one position of start was offered on the app. Several of the participants found this disruptive to their use of the app, as in "split[ing] the exercise routine by routine so that I can start from wherever I want to instead of going through the whole form from routine 1 to 8 ." It was suggested that the video demonstrations "should be continuous but with the ability to stop it if one needs to repeat it. That way, we can do the exercise continuously without having to stop and press the play button." The second most common comment by the participants had to do with the device the app was accessible to. Many comments suggested modifying the Qi Gong Wellness App for Android, PC or Samsung users.

\section{Discussion}

The piloting of the Qi Gong Wellness App extends emerging literature on mobile technology for health and positive longevity for older adults. Highly aware of the growing prevalence of dementia in Canada's aging population, this participant group valued the simplicity of use to incorporate physically modified yet philosophically sound principals of Qi Gong into their routines with 91.67 percent answering affirmatively.

The design of the exercise videos was embraced by 82 percent, reflecting current literature advocating short segments of exercise that are self determined by the user. The underlying premise of "anywhere, anytime" exercise enables the older adult the freedom surrounding fixed scheduling and avoids the potentially costly decision to join organized exercise programs such as those offered by a gym. The provision of mobile wellness app for this population also reflects Canada's health movement toward self management of chronic illnesses.

A key finding from the pilot challenges the stereotypical view that the older adult is fearful of technology and unlikely to consider mobile exercises offered in an app. Rather, our findings support emerging literature that demonstrates the design, utility and user accessibility of an app is well accepted by the older adult, such as the Qi Gong Wellness App, that combines the Eastern art of wellness exercise with Western mobile technology. In fact, this group of 
participants offered technologically informed modifications to the app reflecting a higher level of user knowledge than anticipated by the authors.

A limitation of the study is the assumed inherent positive value toward exercise and aging by the group of participants who volunteered for the pilot. However, the intrinsic motivation to exercise transcends gender, age and education and so it is assumed that the Qi Gong Wellness App would be attractive to those who did not attend the Qi Gong Chinese Health Qi Gong Association Conference.

This pilot project lends rigor and dimension to the little researched area linking mobile technology, cognitive impairment and exercises tailored to older adults who are interested in self monitoring of their physical and cognitive health as protective factors for healthy aging. The Qi Gong Wellness App offers an innovative fusion of eastern philosophy and western technology in response to an aging population invested in physical and cognitive longevity.

\section{References}

Armas, J., Broderick, A., Coye, M., DeMello, S., and Harvath, B. (2009) Technologies to help older adults maintain independence: Advancing technology adoption. Center for Technology and Aging.

British Columbia Ministry of Health (BC) (2014) Senior's Fall Prevention, www.health.gov.bc.ca/prevention/fallprevention.html.

Burgener, S. C., Yang, Y., Gilbert, R., \& Marsh-Yant, S. (2008). The effects of a multimodal intervention on outcomes of persons with early-stage dementia. American journal of Alzheimer's disease and other dementias, 23(4), 382-394.

Cavill, M. (2013) British Heart Foundation Health Promotion Research Group www.nhs.uk/Livewell/fitness/Pages/activities-for-the-elderly.aspx 
Centers for Disease Control and Prevention (2009) Prevalence and most common causes of disability among adults-United States, 2005. Morbidity and Mortality Weekly Report, 58 (16), 421-426.

Center for Technology and Aging (2009) The New Era of Connected Aging: A Framework for Understanding Technologies that Support Older Adults in Aging - 2014 Report Update. www.techandaging.org/ConnectedAgingFramework.pdf

Chinese Health Qigong Association (2013). Chinese Health Qigong. Beijing, Foreign Language Press.

Cohen, K. (1999) The way of qigong: The art and science of Chinese energy healing. Random House LLC.

Dechamps, A., Diolez, P., Thiaudiere, E., Tulon, A., Onifade, C., Vuong, T., Helmer, C. and Bourdel-Marchasson (2010) Effects of exercise programs to prevent decline in health-related quality of life in highly deconditioned institutionalized elderly persons: a randomized controlled trial. Archives of Internal Medicine, 170 (2): 162-169.

Dong, L., Lee, J. B., Kim, Y. K., and Kim, Y. S. (2013) The Effects of Health Qigong Training of Elderly Single Women on Pain Consciousness and Depression. International Journal of Applied Sports Sciences, 25(2): 152-166.

Hopkins, R. (2010) Geriatric Psychiatry Programme, Clinical/Research Bulletin, Number 16. 
Hse, H., Wang, C., Chen, Y., Chang, M. and Wang (2010) Evaluation of a Community Based Aging Intervention Program. Educational Gerontology, 36 (7), 547-572.

Jahnke, R. (2002) The Healing Promise of Qi: Creating Extraordinary Wellness through Qigong and Tai Chi. Chicago, II: Contemporary Books.

Jahnke, R., Larkey, L., Rogers, C., Etnier, J., and Lin, F. (2010) A comprehensive review of health benefits of qigong and tai chi. American Journal of Health Promotion, 24 (6), e1-e25.

Kramer, A. (2010) Dementia projections for the counties, regional municipalities and census divisions of Ontario - Clinical Research Bulletin no. 16. PCCC Mental Health Services, Ontario.

Kwak, Y., Um, T. \& Son, D. (2008) Effect of regular exercise on senile dementia patients. International Journal of Sports Medicine, 29 (6), 471-474.

Larkey, L., Jahnke, R., Etnier, J., and Gonzalez, J. (2009) Meditative movement as a category of exercise: Implications for research. Journal of Physical Activity \& Health. 6: 230-238.

Melenhoust, A., Rogers, W., and Caylor, E. (2001) The use of communication technologies by older adults: Exploring the benefits from the user's perspective. Proceedings of the Human Factors and Ergonomics Society $46^{\text {th }}$ Annual Meeting. Santa Monica, CA: Human Factors and Ergonomics Society.

Rogers, W., and Mynatt, E. (2003) How can technology contribute to the quality of life of older adults? In M.E. Mitchell (Ed.). The technology of humanity: Can technology contribute to the quality of life? (pp. 22-30) Chicago, III: Illinois Institute of Technology. 
Salzman, B.S. (2010) Gait and Balance Disorders in Older Adults, American Family Physician, July 82(1): 61-68

Sancier, K. M., and Holman, D. (2004) Commentary: Multifaceted health benefits of medical qigong. The Journal of Alternative \& Complementary Medicine, 10(1), 163-165.

Smith, A. (2014) Older Adults and Technology

www.pewinternet.org/2014/04/03/older-adults-and-technology-use/

Traywick, L. (2014) Exercise recommendations for older adults.

www.todaysgeriatricmedicine.com/news/ex 092210 03.shtml

Verghese, J., LeValley, A., Hall, C., Katz, M., Ambrose, A., and Lipton, R. (2006) Epidemiology of gait disorders in community-residing older adults. Journal of American Geriatric Society 54(2):255-261.

Wilkson, P. (2010) Rising tide of dementia projected to cost Canada \$872 billion over next 30 years. Media and Government relations - Alzheimer Society of Canada www.alzheimer.ca/ /media/Files/national/Advocacy/ASC Rising Tide Full Report e.pdf 
